\title{
Análisis de las medidas correctoras para la disminución de los eventos adversos en una unidad de hemodiálisis hospitalaria
}

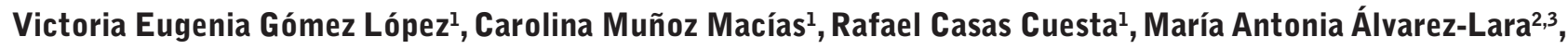 \\ Rodolfo Crespo Montero ${ }^{1,3,4}$ \\ ${ }^{1}$ Servicio de Nefrología. Hospital Universitario Reina Sofía de Córdoba. España \\ ${ }^{2}$ Servicio de Documentación Clínica y Calidad. Hospital Universitario Reina Sofía de Córdoba. España \\ ${ }^{3}$ Departamento de Enfermería. Universidad de Córdoba. España \\ ${ }^{4}$ Instituto Maimónides de Investigación Biomédica de Córdoba. España
}

\section{Resumen}

Los pacientes en hemodiálisis son una población susceptible de padecer eventos adversos por fallos en el proceso asistencial.

Objetivo: Evaluar la eficacia de las medidas correctoras en un plan de seguridad del paciente en hemodiálisis para disminuir los eventos adversos.

Material y Método: Estudio descriptivo y retrospectivo del registro de hemodiálisis de una unidad hospitalaria. Como método de evaluación se utilizó la metodología propia de nuestro centro (MIDEA) basado en el método Global Trigger Tools.

Se revisaron todas las sesiones de hemodiálisis de marzo, mayo y julio de 2016: 2.080 sesiones; y los mismos meses en 2017: 1953 sesiones.

Después del análisis de 2016, se implantaron las siguientes medidas correctoras: revisión del procedimiento de anticoagulación del circuito extracorpóreo, actualización del manejo de los accesos vasculares y actualización del procedimiento ante hipotensiones.

\section{Correspondencia:}

Victoria E. Gómez López

UGC Nefrología, Unidad de Diálisis. Hospital U. Reina Sofía Avda. Menéndez Pidal, s/n. 14004 Córdoba E-mail: morenamia47@gmail.com
Resultados: En el año 2016, se revisaron las gráficas de 208 pacientes. Se detectaron 255 eventos adversos ( $11,8 \%$ de las sesiones), siendo los más frecuentes: 85 hipotensiones, 74 coagulaciones del circuito sanguíneo, 31 problemas del acceso vascular y 65 otros eventos adversos.

En el año 2017, se revisaron las gráficas de 258 pacientes. Se detectaron 155 eventos adversos (7,9\% de las sesiones), siendo los más frecuentes: 60 hipotensiones, 36 coagulaciones del circuito, 30 problemas del acceso vascular y 29 otros eventos adversos.

Conclusiones: Los eventos adversos disminuyeron un $39,2 \%$ en 2017 , y aunque siguen siendo las hipotensiones, coagulaciones del circuito sanguíneo y los problemas derivados del acceso vascular los más frecuentes, pensamos que las medidas correctoras están siendo eficaces.

PALABRAS CLAVE: seguridad del paciente; evento adverso, hemodiálisis.

\section{Analysis of corrective measures for reducing adverse events in a hospital dialysis unit}

\section{Abstract}

Patients on hemodialysis (HD) are a population susceptible to suffering adverse events (AD) due to failures in the healthcare process. 
Objective: To evaluate the efficacy of corrective measures in a HD patient safety plan to reduce AD.

Material and Method: Descriptive and retrospective study of the HD records of a hospital unit. As an evaluation method, we used the methodology of our center (MIDEA) based on the Global Trigger Tools method.

All the HD sessions of March, May and July 2016 were reviewed: 2,080 sessions; and the same months in 2017: 1953 sessions.

After the 2016 analysis, the following corrective measures were implemented: revision of the anticoagulation procedure of the extracorporeal circuit, updating of the vascular access management and updating of the procedure before hypotension.

Results: In 2016, the clinical records of 208 patients were reviewed. 255 EA were detected $(11.8 \%$ of the sessions). The most frequent $A E s$ were: 85 hypotension, 74 blood circuit coagulations, 31 vascular access problems and 65 other AD.

In 2017, the clinical records of 258 patients were reviewed. $155 \mathrm{AE}$ were detected (in $7.9 \%$ of the sessions). The most frequent $\mathrm{AEs}$ were: 60 hypotension, 36 circuit coagulations, 30 vascular access problems and 29 other AE.

Conclusions: Adverse events decreased by $39.2 \%$ in 2017, and although hypotension, blood circuit coagulation and vascular access problems are the most frequent, we consider that corrective measures are being effective.

KEYWORDS: safety; hemodialysis; adverse event.

\section{Introducción}

La seguridad del paciente es un componente esencial de la calidad asistencial, que preocupa y mucho, tanto a los responsables de los servicios sanitarios como a todos los profesionales que intervienen en el proceso asistencial, por las graves repercusiones que pueden tener los fallos de seguridad en la gestión del sistema sanitario, y sobre todo en la salud del paciente ${ }^{1,2}$.

De hecho, en nuestro país y promovidos por el Ministerio de Sanidad, se han realizado distintos estudios para valorar la seguridad del paciente, midiendo la preva- lencia de eventos adversos ( $E A)$, ocurridos tanto en el ámbito asistencial de los hospitales ${ }^{3}$, como en el de atención primaria ${ }^{4}$, con el objetivo de identificar áreas de mejora en la actividad sanitaria.

No obstante, no existe mucha bibliografía sobre EA en hemodiálisis (HD), teniendo en cuenta que las unidades de diálisis, son terreno propicio para los fallos y errores, debido a su dependencia de la tecnología y al perfil actual del paciente (pluripatológico, edad avanzada) 5 .

Otro problema añadido, es que tradicionalmente los equipos clínicos y los directivos de los servicios de salud han intentado detectar los EA, y en general los incidentes de seguridad usando métodos de notificación voluntarios, aunque la realidad, es que se calcula que solo el 10 a $20 \%$ de los errores son reportados y, de ellos, el 90 a 95 por ciento no causan daño a los pacientes ${ }^{6}$. Por tanto, es obvio que se precisa de un modo más efectivo para identificar eventos que causen daño a los pacientes, para cuantificar el grado y gravedad del daño, y para intentar realizar cambios enfocados a disminuir el daño6.

En nuestra experiencia, cuando elaboramos e implantamos en 2011 nuestro Plan de Seguridad del Paciente en Hemodiálisis ${ }^{7}$, con el fin de identificar, conocer las causas y prevenir la aparición de EA, nos encontramos con resultados poco alentadores en la comunicación voluntaria y anónima de los EA.

Es por ello, por lo que en 2016, con el apoyo metodológico de la Unidad de Calidad y Documentación Clínica, realizamos una autoauditoria de los registros de HD, mediante muestreo aleatorio, para la detección de EA, utilizando una metodología propia de nuestro Hospital, denominada Medición de la Incidencia de Eventos Adversos (MIDEA) ${ }^{8,9}$ basado en el método Global Trigger Tools ${ }^{10,11}$, que permite, con un coste aceptable, establecer la incidencia y la naturaleza de los EA, y hacer evaluaciones sistemáticas y regulares en el tiempo.

Estos primeros resultados nos permitieron detectar que los EA se producían en torno al $10 \%$ del total de las sesiones de HD, teniendo en cuenta que el análisis se llevó a cabo tanto en pacientes de un centro periférico, como de una unidad hospitalaria ${ }^{12}$. Además, estos resultados permitieron identificar aquellos EA más frecuentes, y por tanto, sobre los que había que actuar en primer lugar, para intentar disminuir su frecuencia. 
Después de la presentación de los resultados a todo el grupo, se elaboraron una serie de medidas correctoras (procedimientos de actuación) a implantar a principios de 2017, para posteriormente evaluar su eficacia.

Por todo ello, y con la finalidad última de mejorar la seguridad del paciente en nuestros pacientes en HD, nos planteamos como objetivo principal evaluar la eficacia de las medidas correctoras del plan de seguridad implantado en nuestra unidad para disminuir la incidencia de EA.

\section{Material y Método}

Se realizó un estudio observacional descriptivo y retrospectivo en dos fases. En 2016 se realizó una auditoria de los EA registrados y después de la implantación de unas medidas correctoras dentro del Plan de Seguridad del paciente en $\mathrm{HD}$, en la Unidad de Diálisis Hospitalaria del Hospital Universitario Reina Sofía de Córdoba ${ }^{13}$, se comparó la incidencia de EA entre ambas fases, de acuerdo al listado de EA de este Plan (Tabla 1). Los EA $8,10,15$ y 16 se agruparon como Problemas del acceso vascular (AV). Se recogieron además, los datos demográficos de los pacientes: edad, tipo de paciente (agudo o crónico) y tipo de acceso vascular (AV).

Tabla 1. Listado de Eventos Adversos. Plan de Seguridad del Paciente en Hemodiálisis.

\begin{tabular}{ll}
\hline 1 & Transmisión de enfermedades víricas \\
\hline 2 & Hipotensión \\
\hline 3 & Síndrome de desequilibrio en primeras diálisis \\
\hline 4 & Coagulación del sistema \\
\hline 5 & Embolia gaseosa \\
\hline 6 & Calambres \\
\hline 7 & Hemólisis \\
\hline 8 & Infección del acceso vascular \\
\hline 9 & Hemorragias/hematomas sistémicos \\
\hline 10 & Rotura y/o salida del catéter \\
\hline 11 & Errores en la administración de medicación \\
\hline 12 & Inadecuado tratamiento dialítico \\
\hline 13 & Reacción a pirógenos \\
\hline 14 & Caída durante la diálisis \\
\hline 15 & Salida de aguja durante la diálisis \\
\hline 16 & Sangrado excesivo de los puntos de punción \\
\hline 17 & Error en la petición de analítica \\
\hline
\end{tabular}

En la primera fase de estudio se revisaron todas las sesiones de HD de los meses de Marzo, Mayo y Julio de 2016. Se revisaron las gráficas de HD de 2.080 sesiones, correspondientes a 208 pacientes (17\% agudos, 83\% crónicos), con una edad media de 67 años (15-93). Se revisaron una media de 10 sesiones/paciente (1-66). EI AV de los pacientes revisados tenía la siguiente distribución: 117 pacientes tenían una Fístula Arteriovenosa (FAV), 48 un Catéter Venoso Central Tunelizado y 45 un Catéter Venoso Central no Tunelizado.

Una vez analizados los datos de 2016, estos fueron presentados a todos los profesionales de la Unidad de Diálisis, en reunión de Formación en Servicio, para conocer los resultados y sobre todo, para analizar cuáles habían sido los EA más prevalentes. En esta reunión se hizo especial hincapié en el carácter informativo y de búsqueda de mejora, y no punitivo, de estos resultados.

A continuación se programaron otras Reuniones en las que se abordaron las Revisiones de los procedimientos que presentaban mayor frecuencia de EA (Hipotensión, coagulaciones del circuito extracorpóreo y problemas relacionados con el $\mathrm{AV}$ ):

- Modificación e implantación del procedimiento de anticoagulación del circuito sanguíneo extracorpóreo.

- Revisión del procedimiento de actuación ante las hipotensiones.

- Revisión y actualización del manejo de los accesos vasculares, de acuerdo a la Guía Clínica Española del Acceso Vascular de Hemodiálisis de $2016^{14}$.

Las modificaciones de estos procedimientos específicos se fueron implantando tras la presentación de los mismos (Último trimestre de 2016), incidiendo en establecer las medidas encaminadas a prevenir estos EA más prevalentes.

En la segunda fase del estudio, en 2017, se revisaron todas las sesiones de HD de los mismos meses que en la fase anterior (marzo, mayo y julio). Se revisaron las gráficas de HD de 1953 sesiones, correspondientes a 258 pacientes ( $13 \%$ agudos, $87 \%$ crónicos), con una edad media de 67 años (15-93). Se revisaron una media de 7,5 sesiones/paciente (1-43). EI AV de los pacientes revisados tuvo la siguiente distribución: 142 pacientes tenían una FAV, 74 un Catéter Venoso Central Tunelizado y 42 un Catéter Venoso Central no Tunelizado. 
Para la autoauditoria, se utilizó el instrumento MIDEA ${ }^{8,9}$ basado en el método Global Trigger Tools $^{10}$, consistente en un análisis estructurado, sistemático y exhaustivo de los registros de enfermería de HD, y para evitar sesgos de interpretación, estas revisiones fueron llevados a cabo por dos enfermeras, entrenadas al efecto, que fueron las mismas en las dos fases de estudio.

Los EA detectados, se categorizaron por su gravedad según la escala establecida por la National Coordinating Council for Medication Error Reporting and Prevention (NCC MERP) ${ }^{15}$, registrándose finalmente solo los $E A$ que describen daño al paciente (categorías $E, F, G, H$ e I), y que precisaron intervención.

Se realizó un análisis descriptivo de las variables cualitativas y cuantitativas, en una hoja de cálculo del Excel 2016 (Microsoft).

\section{Resultados}

En 2016 se registraron 255 EA en 2080 sesiones revisadas, es decir hubo un $11,8 \%$ de EA en todas las sesiones ( 1 EA cada 8,1 sesiones). Estos EA fueron registrados en las gráficas de 208 pacientes (1,2 EA por paciente): 127 pacientes no tuvieron ningún EA y 61 pacientes tuvieron al menos 1 EA.

En 2017 se registraron 155 EA en 1953 sesiones revisadas, lo que supuso que en el $7,9 \%$ de las sesiones hubo EA (1 EA cada 12,6 sesiones). Estos EA fueron registrados en las gráficas de 258 pacientes $(0,6$ EA por paciente): 167 pacientes no tuvieron ningún EA y 93 pacientes tuvieron al menos 1 EA.

Al comparar la incidencia global de EA entre ambos años, encontramos una disminución importante en 2017 respecto a 2016 (255 frente a 155) lo que supone una reducción del 39,2\% de EA en 2017.

En la Tabla 2 se muestran la distribución de los EA por año, en número absoluto y su proporción respecto al número total.

Respecto a la disminución de EA más prevalentes y objeto de las medidas correctoras, entre los años comparados podemos observar en la Figura 1, como ha habido una disminución en todos los EA, y muy especialmente en las coagulaciones del circuito extracorpóreo y las hipotensiones.
Tabla 2. Comparación en el número total de EA entre el año 2016 y 2017.

\begin{tabular}{lcccc}
\hline & 2016 & $\%$ del total & 2017 & \% del total \\
\hline Hipotensiones & 85 & $33,3 \%$ & 60 & $38,7 \%$ \\
Coagulaciones circuito & 74 & $29 \%$ & 36 & $23,2 \%$ \\
Problemas AV & 31 & $12,1 \%$ & 30 & $19,3 \%$ \\
Otros EA & 65 & $25,4 \%$ & 29 & $18,7 \%$ \\
\hline Total EA registrados & 255 & & 155 & \\
\hline
\end{tabular}

En otros EA registrados se han agrupado el resto de EA.

Respecto a la categorización de los EA, según su Gravedad (escala NCC MERP), y que corresponden a EA que han originado daños temporales al paciente y han requerido intervención, en 2016 se encontraron 222 EA de Categoría E (Error que puede haber contribuido o resultado en daño temporal al paciente y que requiere intervención) y 16 de Categoría $\mathbf{F}$ (Error que puede haber contribuido o resultado en daño temporal al paciente y que requiere iniciar o prolongar el ingreso). En 2017 se encontraron 33 EA de Categoría E y 133 de Categoría F.

\section{Discusión}

Aunque poco a poco, el concepto de seguridad del paciente se va interiorizando en las estructuras de la atención sanitaria, y sobre todo, en la actualidad supone una preocupación de primer orden para los responsables de la gestión y profesionales sanitarios en general $^{1,3,4,16}$.

Pero no sólo en los profesionales de la gestión es una cuestión de primer orden; también en el resto de profesionales se hace cada vez más visible la "cultura de la seguridad"17, adquiriendo cada vez más relevancia en las unidades de diálisis, porque aunque la HD ha evolucionado haciéndose una técnica muy segura y más confortable para el paciente, en gran medida debido al desarrollo tecnológico, todavía se trata de un procedimiento complejo no exento de riesgos, que incluso pueden llegar a ser letales 5 .

Es por lo que, nuestro grupo apostó por el desarrollo de un programa de seguridad del paciente en HD, buscando reducir la aparición de EA en nuestra unidad; y porque estamos convencidos de que los riesgos que creíamos inherentes a la técnica de HD son evitables o se pueden minimizar ${ }^{17}$. 


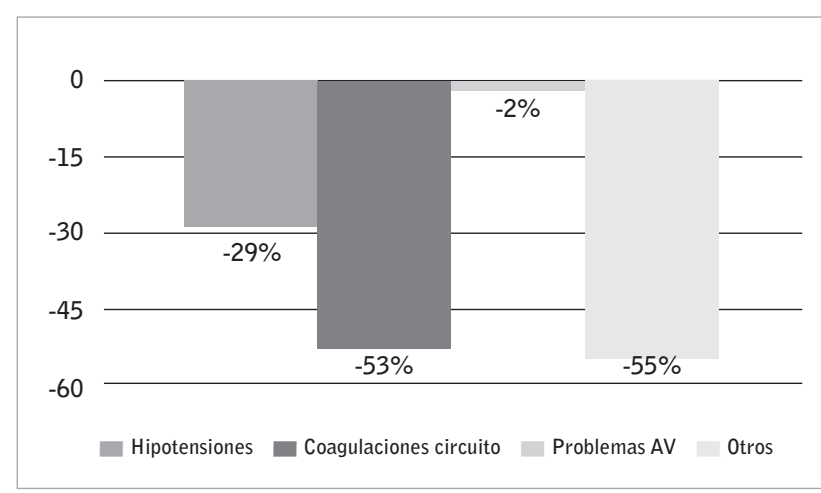

Figura 1. Porcentaje de reducción de EA entre 2016 y 2017.

En efecto, nuestros resultados confirman esta idea, ya que aplicando una serie de medidas correctoras sobre los EA mas prevalentes en la primera auditoria de 2016, hemos conseguido reducir la incidencia de EA en nuestra unidad hospitalaria en torno al $40 \%$ en un año, encontrando 1 EA por cada 12,6 sesiones y 0,6 EA por paciente en 2017 frente a 1 EA cada 8,1 sesiones y 1,2 EA por paciente en 2016, siendo estos resultados inferiores a los encontrados por otros autores de nuestro entorno ${ }^{18,19}$; si bien, se producen después de la aplicación de medidas de mejora. En otro contexto sanitario Holley encuentra una tasa de fallo de 1 cada 733 tratamientos de $\mathrm{HD}^{20}$, la cual es muy baja. Esto es importante de resaltar pues en el momento actual es complicada la comparación de EA encontrados en HD, porque existen definiciones diferentes de la variable objeto de revisión (EA, complicación, fallo), diferentes registros y metodologías de medición; y sobre todo, desconocemos el grado de cumplimentación de los diferentes registros de HD, entre los que no hay uniformidad en su estructura.

Por ello, pensamos que la única forma de comparar tus resultados en este ámbito, es la auto-comparación, es decir ir comparando tus propios resultados a lo largo del tiempo. En nuestro caso, y teniendo en cuenta que independientemente de que haya podido existir defecto de registro de EA, el ámbito, el personal de enfermería, la metodología, las evaluadoras, el periodo, es el mismo en ambos periodos; por lo que cabe inferir que las medidas corrigiendo los procedimientos, y sobre todo, la insistencia en las reuniones de gestión del carácter informativo y de búsqueda de mejora, y no punitivo de este proceso, han ayudado a que todo el grupo tome conciencia de este problema e interiorizar la cultura de la seguridad ${ }^{16}$; de hecho, se ha producido un cambio muy positivo en la mentalidad de los profesionales de enfermería de la unidad, respecto a la rigurosidad en la cumplimentación de los registros.

Respecto los EA más prevalentes, hay que indicar que aunque con una notable mejora entre 2016 y 2017, su distribución es muy parecida en ambos periodos, siendo la hipotensión el EA más frecuente, seguida de la coagulación del circuito extracorpóreo y problemas derivados del $\mathrm{AV}$, en la línea de lo publicado por otros autores de nuestro país ${ }^{18,19}$ y en otros contextos sanitarios $^{21,22}$. En nuestros resultados, las coagulaciones del circuito extracorpóreo fueron la segunda complicación más frecuente, que si bien destacan como frecuente algún estudio ${ }^{19}$, no es destacado por otros autores ${ }^{18,20}$. A este respecto, pensamos que puede ser debido a la particularidad del ámbito en la que se ha desarrollado el estudio: una unidad hospitalaria, en la que además del programa de HD crónicas, asume las diálisis de pacientes agudos, la mayoría con catéteres como $\mathrm{AV}$, y en algunos pacientes, HD con Unipunción, factores todos estos que favorecen la coagulación del sistema, sin olvidar que las actuales unidades de diálisis son organizaciones complejas en las que interaccionan profesionales de diferentes disciplinas y que utilizan tecnología avanzada para tratar pacientes, con frecuencia, de edad avanzada y comorbilidad asociada ${ }^{23}$.

En cuanto a la gravedad de los EA encontrada, según la clasificación NCC MERP15, es importante destacar que no se ha utilizado esta clasificación por otros autores, para clasificar EA en HD y por otra parte que nuestros resultados son muy contradictorios, entre los dos periodos comparados, pues en 2016 se encontraron 222 EA de Categoría E (más leves) y 16 de Categoría $F$, mientras que por el contrario en 2017 se encontraron 33 EA de Categoría E y 133 de Categoría F. Tratándose de las mismas evaluadoras y del mismo escenario asistencial, pensamos que esta diferencia se debe a un error de concepto del grado de gravedad en la primera auditoria (2016), al clasificar los EA de la categoría E (error que puede haber contribuido o resultado en daño temporal al paciente y que requiere intervención); y que la visión más realista de nuestros EA respecto a esta clasificación es la de 2017.

Las principales limitaciones de este estudio han sido que la detección de EA se realiza a través del registro de HD, y que en estos no siempre se registro todo lo que ocurre, además de que no todo el grupo de enfermería tienen claro todos los EA que hay que registrar, pues todos lo que suelen ser complicaciones están registrados, como por ejemplo la Hipotensión, pero en cambio otros 
como Tratamiento dialítico inadecuado puede que estén infra registrados.

A la vista de nuestros resultados podemos concluir que Ios EA disminuyeron en torno al $40 \%$ en 2017 , por lo que podemos considerar que las medidas correctoras están siendo eficaces. Los EA más frecuentes siguen siendo la hipotensión, la coagulación del circuito y los problemas relacionados con los problemas del AV.

Al margen de la eficacia de las medidas correctoras, pensamos que solo por el hecho de monitorizar de forma sistemática los EA y realizar auditorías periódicas, se disminuyen estos EA, lo que nos anima a seguir trabajando en la misma línea, al ser conscientes de que es nuestra responsabilidad y que estamos obligados a intentar reducir la aparición de EA, para garantizar la seguridad del paciente en nuestra unidad.

Recibido: 15 julio 2018

Revisado: 10 agosto 2018

Modificado: 13 septiembre 2018

Aceptado: 17 septiembre 2018

\section{Bibliografía}

1. Aranaz JM, Moya C. Seguridad del paciente y calidad asistencial. Rev Calid Asist. 2011 Dec; 26(6):331-2.

2. Seguridad del paciente. Ministerio de Sanidad, Servicios Sociales e Igualdad [consultado 8 Feb 2018]. Disponible en: http://www. seguridaddelpaciente.es.

3. Ministerio de Sanidad y Consumo. Estudio Nacional sobre los Efectos Adversos ligados a la Hospitalización. ENEAS 2005. [Consultado 10 0ct 2018]. ENEAS 2005. Madrid, 2006. Disponible en: http:// www.mscbs.gob.es/organizacion/sns/planCalidadSNS/pdf/excelencia/opsc_sp2.pdf.

4. Estudio APEAS. Estudio sobre la seguridad de los pacientes en atención primaria de salud. [Consultado 1 0ct 2018]. Madrid: Ministerio de Sanidad

y Consumo; 2008. Disponible en: https://www.seguridaddelpaciente.es/resources/contenidos/castellano/2008/APEAS.pdf.

5. D. Schwappach. Patient safety: What is it all about? Contrib Nephrol, 184 (2015). p. 1-12.

6. 2010 Health Foundation. Research scan: Global trigger tools. [Consultado 10 0ct 2018]. Disponible en: http://www.health.org.uk/public/ cms/75/76/313/2601/Global\%20trigger\%20tools. pdf?real Name $=$ va7dt0.pdf

7. Alcántara M, Martínez A, Guisado C, López MD, Álvarez-Lara MA, Crespo R. Implantación de un plan de seguridad del paciente en hemodiálisis [consultado 30 0ct 2018]. Disponible en: http://www.revistaseden.org/files/3502 Artículo\%20100.pdf.

8. Tejedor $M$, Montero-Pérez FJ, Miñarro R, Gracia-García F, Roig JJ, García-Moyano AM. Diseño e implantación de un plan de seguridad del paciente en un servicio de urgencias de hospital: ¿cómo hacerlo?. Emergencias 2013;25:218-27.

9. Paredes RM, Castillo AL, Miñarro R, Garrido-Pérez, Granero R, Gómez-Beltrán 0 et al. Elaboración de un plan de seguridad del paciente en un Servicio de Cirugía Pediátrica. Cir Pediatr 2014;27:157-64.

10. Griffin FA, Resar RH. IHI Globar Trigger Tool for Measuring Adverse Events (Second Edition). IHI Innovation Series white paper. Cambridge, Massachusetts: Institute for Healthcare Improvement; 2009. [Consultado 10 0ct 2018]. Disponible en: http://www.ihi.org/resources/Pages/IHIWhitePapers/IHIGlobalTriggerToolWhitePaper.aspx

11. Sharek PJ, Horbar JD, Mason W, Bisarya $H$, Thurm CW, Suresh G, et al. Adverse Events in the Neonatal Intensive Care Unit: Development, Testing, and Findings of an NICU Focused Trigger Tool to Identify Harm in North American NICUs. Pediatrics 2006; 118:1332-40.

12. Muñoz Macías $C$, Torollo Luna I, Sag Legrán $M^{a} J$, Salas Cardador F, Gómez López VE, Crespo Montero R. Análisis de los eventos adversos en una unidad de diálisis. Enferm Nefrol 2017;20(Supl 1):S29.

13. Álvarez-Lara MA, Crespo R. López-Andreu M, Berenguer MJ Tejedor M. Programa de Seguridad del 
Paciente. Unidad de Gesión Clínica de Nefrología. V-4. Hospital Universitario Reina Sofía de Córdoba. [Consultado 10 Sept 2018]. Disponible en: https:// www.juntadeandalucia.es/servicioandaluzdesalud/ hrs3/fileadmin/user_upload/area_medica/nefrologia/programa_seguridad_paciente_ugc_nefrologia_ v4_2017.pdf

14. Guía Clínica Española del Acceso Vascular de Hemodiálisis. [Consultado 10 0ct 2016]. Disponible en: https://www.senefro.org/modules.php? name=webstructure \&idwebstructure $=92$

15. NCC MERP Index for categorizing Medication errors. [Consultado 10 Feb 2016]. Disponible en: https://www.nccmerp.org/sites/default/files/indexColor2001-06-12.pdf

16. Tomás S, Chanovas M, Roqueta F, Alcaraz J, Toranzo T y grupo de trabajo EVADUR-SEMES: eventos adversos ligados a la asistencia en los servicios de urgencias de hospitales españoles. Emergencias 2010;22:415-28.

17. Sánchez-Izquierdo FL, Olmedo-Moreno N, Urbón-Peláez N, Rico- González A. Análisis de la cultura de seguridad del paciente en unidades extra-hospitalarias de hemodiálisis. Evolución tras la implantación de medidas. Enferm Nefrol. 2018;21 (1):25-33.
18. Arenas-Jiménez MD, Ferre G, Álvarez-Ude F. Estrategias para aumentar la seguridad del paciente en hemodiálisis: Aplicación del sistema de análisis modal de fallos y efectos (sistema AMFE). Nefrología. Diciembre 2017;37(6):563-70.

19. Matarán-Robles M, Aguilar-García R, Muñoz-Becerra M. Incidencia y tipo de efectos adversos durante el procedimiento de hemodiálisis. Enferm Nefrol. 2013;16(1):36-40.

20. Holley JL. A descriptive report of errors and adverse events in chronic hemodialysis units. Nephrol News Issues. 2006;20:57-8.

21. Mancini A, Angelini P, Bozzi M, Cuzzola C, Giancaspro V, Laraia $\mathrm{E}$. Analysis of clinical risk and adoption of shared procedures: Experience of nephrology and dialysis unit of ASL. G Ital Nefrol. 2015;32:5.

22. Sands JJ, Usvyat LA, Sullivan T, Segal JH, Zabetakis $\mathrm{P}$, Kotanko $\mathrm{P}$, et al. Intradialytic hypotension: Frequency, sources of variation and correlation with clinical outcome. Hemodial Int. 2014;18:415-22.

23. Garrick R, Kliger A, Stefanchik B. Patient and facility safety in hemodialysis: Opportunities and strategies to develop a culture of safety. Clin J Am Soc Nephrol. 2012;7:680-8.

Este artículo se distribuye bajo una Licencia Creative Commons Atribución-NoComercial 4.0 Internacional. https://creativecommons.org/licenses/by-nc/4.0/ 